\title{
Study of the Influence of Reference System in Surface Finishing Parameters Evaluation
}

\author{
A. Sanz ${ }^{(1)}$, R. Fernández ${ }^{(2)}$, S. Pindado ${ }^{(3)}$, P. Núñez ${ }^{(4)}$ \\ ${ }^{(1,2)}$ Department of Aerospace Materials and Production. ETSI Aeronáuticos-UPM. \\ Plaza Cardenal Cisneros 3, 28040 Madrid, Spain, a.slobera@upm.es. \\ (3) University Institute of Microgravity "Ignacio Da Riva". IDR/UPM. ETSI Aeronáuticos-UPM. \\ Plaza Cardenal Cisneros 3, 28040 Madrid, Spain. \\ (4) ETSI Industriales-UCLM, Avda. Camilo José Cela, 3, 13071-Ciudad Real, Spain.
}

\begin{abstract}
In the present paper the influence of the reference system with regard to the characterization of the surface finishing is analyzed. The effect of the reference system's choice on the most representative surface finishing parameters (e.g. roughness average $R a$ and root mean square values $R q$ ) is studied. The study can also be applied to their equivalent parameters in waviness and primary profiles. Based on ISO and ASME standards, three different types of regression lines (central, mean and orthogonal) are theoretically and experimentally analyzed, identifying the validity and applicability fields of each one depending on profile's geometry.
\end{abstract}

Keywords: microgeometry, surface finishing, roughness, waviness, reference lines

\begin{abstract}
RESUMEN
El presente trabajo realiza un estudio de la influencia que supone la elección del sistema de referencia en la determinación los valores de los parámetros más relevantes empleados en la caracterización del acabado superficial tales como la rugosidad media aritmética $R a$ o la rugosidad media cuadrática $R q$ y sus equivalentes en los perfiles de ondulación y completo. Partiendo de la definición establecida por las normas ISO y ASME, se analizan tres tipos de líneas de regresión cuadrática (línea central, línea media y línea ortogonal), delimitando los campos de validez y de aplicación de cada una de ellas en función de la geometría del perfil. Para ello se plantean diversos tipos de perfiles y se desarrolla un estudio teórico y experimental de los mismos.
\end{abstract}

Palabras clave: microgeometría, acabado superficial, rugosidad, ondulación, líneas de referencia

\section{Introducción}

El acabado superficial, entendido como el estudio de las propiedades microgeométricas de las superficies de las piezas y de su influencia en el comportamiento funcional de las mismas, reviste una gran importancia en la ingeniería de los procesos de fabricación, debido a la gran variedad de comportamientos funcionales que se ven afectados por él, algunos de los cuales se esquematizan en la figura 1.

Sin menoscabo de otras aplicaciones, las técnicas más ampliamente difundidas en la medición y caracterización del grado de acabado superficial, se basan en la definición de un perfil obtenido a partir de la información de medida proporcionada por el rugosímetro con palpador inductivo de contacto. La delimitación de la zona de medida y la elección del sistema de referencia en el perfil son factores clave en la determinación de los valores de parámetros más representativos empleados en la caracterización del acabado superficial ya que permiten la intercomparación de mediciones realizadas en distintas zonas de la superficie o en diferentes instrumentos de medida asegurando la repetibilidad de los resultados.

La importancia tecnológica asociada al estudio y la caracterización del acabado superficial queda de manifiesto en la consideración que el tema recibe en la mayoría de la bibliografía relacionada con los procesos de fabricación. 


\section{Tribología}

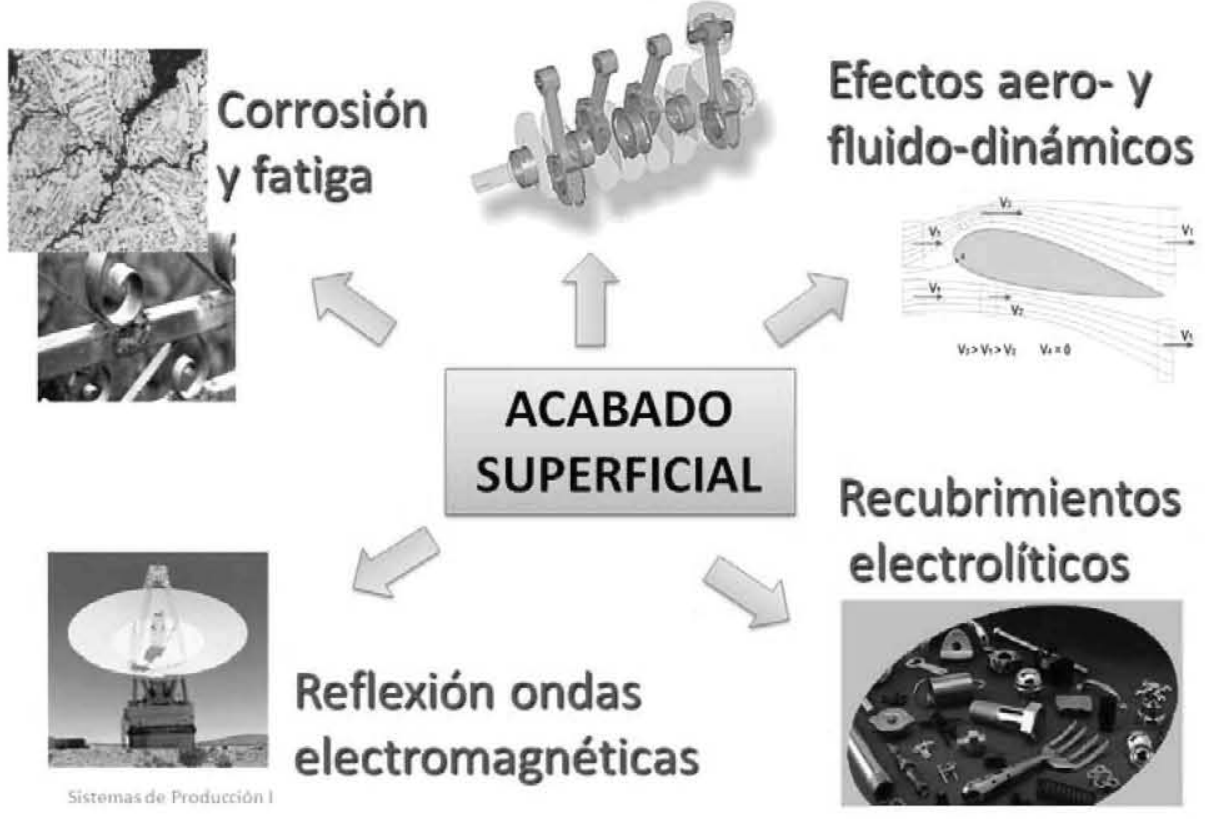

Figura 1. Comportamientos funcionales influenciados por el acabado superficial

En algunos casos el acabado superficial se aborda de forma monográfica [1] mientras que en otros textos de carácter más generalista se aborda en algunos de sus capítulos [2]. También la normativa nacional e internacional refleja la importancia del estudio del acabado superficial. Existe una amplia variedad de normas relativas entre las que cabe destacar las elaboradas por ISO [3] y por ASME [4] a las cuales se supeditan la mayor parte de las normas nacionales. Dichas normas han experimentado diversas revisiones a lo largo de los últimos años. Este hecho pone de manifiesto la actualidad del tema y la constante búsqueda de mejoras en la definición, medición y caracterización del acabado superficial.

Para caracterizar el acabado superficial, existen diversos parámetros, casi todos ellos numéricos, siendo la rugosidad media aritmética $R a$ y la rugosidad media cuadrática $R q$ los dos más ampliamente difundidos. Todos los parámetros requieren la delimitación de la zona en la que se evalúan así como las características de filtrado de la información empleada en su determinación, ya que de lo contrario, los resultados obtenidos no son comparables entre sí. El presente trabajo se centra en la definición del sistema de referencia respecto a la cual se obtienen algunos de los parámetros más representativos tales como los ya citados $R a$ y $R q$.

\section{Sistema de referencia}

De una manera simplificada, tal y como esquematiza la figura 2, puede decirse que el rugosímetro mecánico recibe desde la unidad de palpado una señal proporcional a los desplazamientos (eje $z$ ) relativos de la punta de contacto respecto a la trayectoria que sigue en su desplazamiento el cabezal de medida (eje $x$ ). Dicha señal es procesada y filtrada internamente y se convierte en una serie de valores digitales $\left(x_{i}, z_{i}\right)$ correspondientes a los perfiles completo $(P)$, de rugosidad $(R)$ o de ondulación $(W)$ a partir de los cuales se determinan los parámetros que caracterizan el acabado superficial. Los rugosímetros realizan de forma interna las operaciones necesarias para obtener los parámetros sin que exista posibilidad por parte del usuario de interaccionar en dicho proceso de cálculo. De hecho, si no se dispone de la información numérica de los valores que constituyen el perfil no queda más remedio que dar por buenos los resultados presentados e implícitamente el método de cálculo utilizado. Tal y como se verá a continuación, la elección del sistema de referencia puede producir variaciones significativas en los resultados obtenidos, dependiendo de la forma del perfil. La determinación del sistema de referencia requiere la definición de la dirección de los ejes $z$ y $x$, la definición del origen y la definición del intervalo que será objeto de estudio. El presente trabajo se centra en la posición de los ejes $z$ y $x$ y del origen, asumiendo que la longitud en la que se determinan los valores de los parámetros es la requerida en caso conforme a la norma (longitud básica o longitud de medición). 


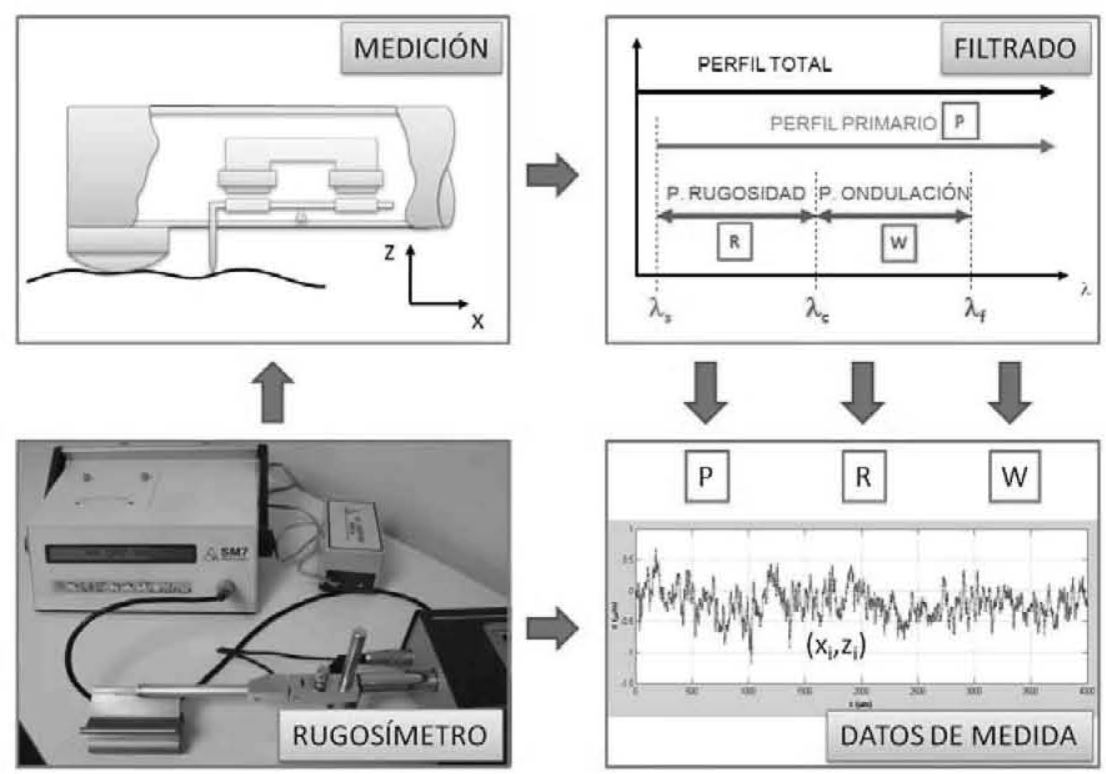

Figura 2. Esquema de obtención de los datos de un perfil

Cuando se dispone de la información digitalizada $\left(x_{i}, z_{i}\right)$ correspondiente a uno cualquiera de los perfiles considerados, tanto las normas ISO como la norma ASME definen el eje $x$ a partir del concepto de línea media, entendida como una línea de mínimos cuadrados con la misma forma que la línea de referencia del perfil nominal o teórico. Por lo general, dicha línea es una línea recta obtenida imponiendo la condición de mínimos cuadrados a las distancias entre los puntos del perfil y la recta.

El término "línea de mínimos cuadrados" presenta cierta ambigüedad ya que existen diversas posibilidades que tienen cabida dentro de su ámbito. Entre las diferentes líneas de mínimos cuadrados existentes se van a considerar las tres indicadas en la Tabla I.

Tabla I. Líneas de mínimos cuadrados

\begin{tabular}{cccc}
\hline Denominación común & Minimiza & Pendiente & Abreviatura \\
\hline Línea central & Alturas $\left(z_{i}\right)$ & $\mathrm{m}=0$ & $L C$ \\
Línea media & Alturas $\left(z_{i}\right)$ & $\mathrm{m} \neq 0$ & $L M$ \\
Línea ortogonal & Distancias $\left(\sqrt{x_{i}^{2}+z_{i}^{2}}\right)$ & $\mathrm{m} \neq 0$ & $L O$ \\
\hline
\end{tabular}

La línea central $L C$ es la línea horizontal de mínimos cuadrados. Su determinación es la más sencilla ya que su pendiente $m$ es nula y las distancias de los puntos del perfil a ella coinciden con las ordenadas $z_{i}$ de los puntos. La ecuación de la recta respecto al sistema de los valores medidos es de la forma $z=b$, pudiendo determinarse el valor de $b$ mediante la expresión

$$
b=\frac{1}{n} \sum_{i=1}^{n} z_{i}
$$

donde $n$ representa el número de puntos del perfil digitalizado.

La línea media $L M$ minimiza la suma de los cuadrados de las distancias verticales de los puntos a ella. A diferencia de la línea central, la línea media no tiene porqué ser paralela al eje de abscisas. Su ecuación es de la forma $z=m x+b$, pudiendo determinarse los coeficientes $m$ y $b$ a partir de las expresiones

$$
m=\frac{n \sum_{i=1}^{n} x_{i} z_{i}-\left(\sum_{i=1}^{n} x_{i}\right)\left(\sum_{i=1}^{n} z_{i}\right)}{n \sum_{i=1}^{n} x_{i}^{2}-\left(\sum_{i=1}^{n} x_{i}\right)^{2}} ; b=\frac{1}{n} \sum_{i=1}^{n} z_{i}-m \frac{1}{n}\left(\sum_{i=1}^{n} x_{i}\right)
$$


Finalmente, la línea ortogonal $L O$ es la línea de mínimos cuadrados que hace mínima la suma de los cuadrados de las distancias de los puntos a ella. Al igual que la línea media no tiene porqué ser paralela al eje de abscisas. Pese a ser la línea que minimiza la suma de cuadrados de distancias, su utilización es la menos difundida de las tres líneas consideradas. Los coeficientes $m$ y $b$ de su ecuación $z=m x+b$. pueden determinarse a partir de las expresiones

$$
m=\frac{-u \pm \sqrt{u^{2}+4 v^{2}}}{2 v} \quad ; \quad b=\frac{1}{n} \sum_{i=1}^{n} z_{i}-m \frac{1}{n} \sum_{i=1}^{n} x_{i}
$$

en las que $u$ y $v$ se determinan mediante

$$
\begin{gathered}
u=\frac{1}{n}\left(\sum_{i=1}^{n} x_{i}^{2}-\sum_{i=1}^{n} z_{i}^{2}\right)-\frac{1}{n^{2}}\left(\left(\sum_{i=1}^{n} x_{i}\right)^{2}-\left(\sum_{i=1}^{n} z_{i}\right)^{2}\right) \\
v=\frac{1}{n} \sum_{i=1}^{n} x_{i} z_{i}-\frac{1}{n^{2}}\left(\sum_{i=1}^{n} x_{i}\right)\left(\sum_{i=1}^{n} z_{i}\right)
\end{gathered}
$$

Para poner de manifiesto la repercusión que en el valor final de los parámetros puede tener la elección de uno u otro tipo de línea supóngase un perfil formado por triángulos isósceles tal y como el representado en la figura 3. Dicho perfil solamente se plantea como elemento de apoyo a la exposición ya que no se corresponde con una situación real de medida al ser los valores de sus alturas muy superiores a los que pueden aparecer en cualquier pieza.

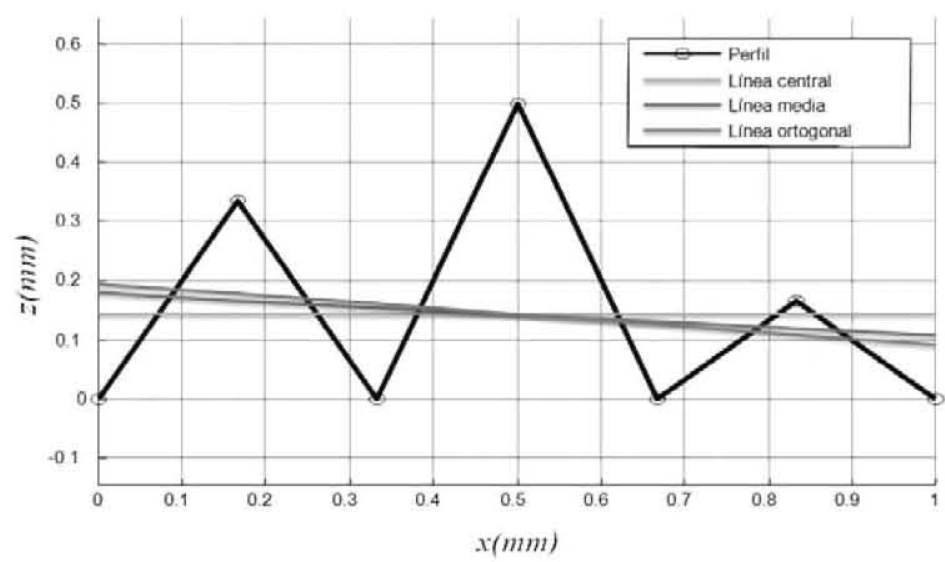

Figura 3. Perfil de ejemplo

La determinación de los valores de $R a$ y $R q$ en función de las tres líneas consideradas conduce a los valores recogidos en la Tabla II.

Tabla II. Valores calculados para el perfil de ejemplo (Figura 3)

\begin{tabular}{cccc}
\hline Línea & Ecuación & $\boldsymbol{R a}$ & $\boldsymbol{R} \boldsymbol{q}$ \\
\hline $\boldsymbol{L C}$ & $z=0.14286$ & 0.16327 & 0.18748 \\
$\boldsymbol{L O}$ & $z=-0.10336 x+0.19454$ & 0.16240 & 0.18528 \\
$\boldsymbol{L M}$ & $z=-0.071429 x+0.17857$ & 0.16285 & 0.18549 \\
\hline
\end{tabular}

Puede observarse como los valores de los parámetros referidos a las líneas $L M$ y $L O$ apenas presentan variación, mientras que en relación a los referidos a $L C$ aparece una variación mayor. También puede observarse que el valor asociado a $L O$ es menor que el de $L M$ y $L C$. Aun tratándose de un ejemplo particular, este hecho hace pensar que para una misma medición de acuerdo con lo establecido en las normas ISO y ASME la elección de la $L O$ como línea de referencia conduce a unos valores menores de los parámetros $R a$ y $R q$. 


\section{Metodología}

Para determinar el orden de magnitud de la diferencia existente entre los valores de los parámetros calculados referidos a uno u otro tipo de línea es importante tener en consideración la relación entre la altura máxima del perfil y su longitud. En efecto, llamando $H$ a la altura máxima del perfil y $L$ a longitud en la que se evalúan los parámetros, cuanto mayor sea la relación $H / L$ mayor es la diferencia que supone la elección de uno u otro tipo de línea, mientras que para valores de $H / L$ pequeños, dicha diferencia se reduce pudiendo llegar a ser despreciable.

\subsection{Influencia del parámetro $H / L$ en la pendiente de las líneas de mínimos cuadrados}

Utilizando un perfil con la misma forma que el perfil de ejemplo representado en la Figura 3, puede observarse que si se varía el parámetro $H / L$, la pendiente de la línea ortogonal y la de la línea media experimentan una variación tal y como la representada en la Figura 4. Se observa que cuando la coordenada $x$ es dominante frente a la $z$ (valores pequeños de $H / L$ ) la pendiente de ambas rectas en valor absoluto tiende a cero, mientras que cuando la $z$ es dominante (valores grandes de $H / L$ ) la pendiente en valor absoluto aumenta considerablemente.

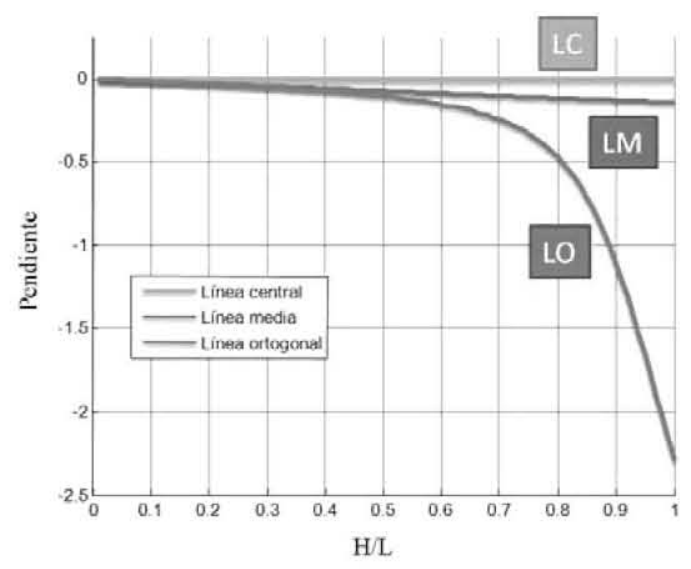

Figura 4. Pendiente vs relación $H / L$ en las líneas de referencia

Representando la diferencia máxima en la coordenada $z$ de la línea media y la línea ortogonal, se obtiene el resultado mostrado en la Figura 5. En ella puede apreciarse como la diferencia entre ambas líneas decrece a medida que lo hace el parámetro $H / L$.

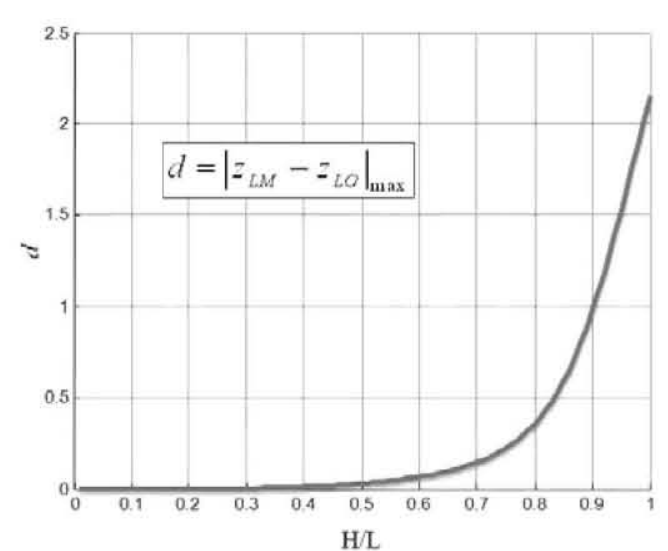

(a)

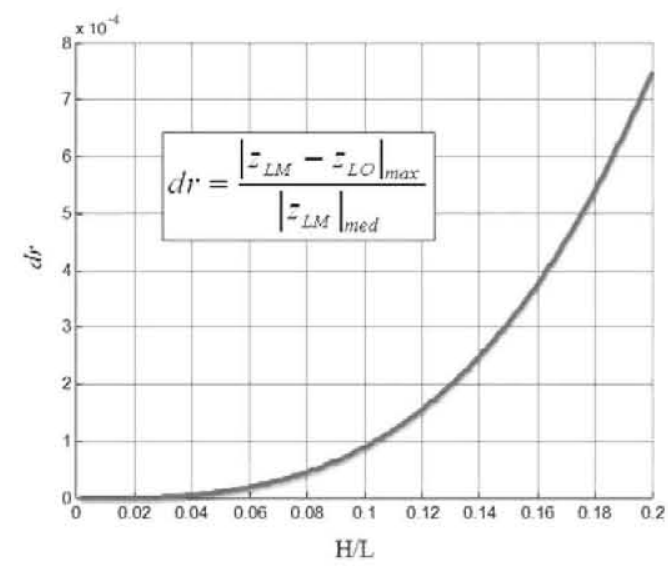

(b)

Figura 5. Diferencias absolutas y relativas entre las ordenadas de las lineas LM y LO

En la Figura 5(b) se presenta un estudio más detallado de la región $H / L<0,2$. Para dejar patente que estas diferencias son muy pequeñas se ha optado por una representación adimensional de esta diferencia empleando el máximo valor de la coordenada $z$ de la línea media para la adimensionalización. 


\subsection{Fundamento matemático}

Aunque los resultados presentados se han obtenido trabajando sobre una geometría de perfil particular, el comportamiento mostrado por la pendiente de ambas líneas frente a la relación $H / L$ es cualitativamente semejante al descrito. En efecto, si se comparan las expresiones matemáticas cuya minimización conduce a cada tipo de línea, se tiene que en el caso de la línea media la función a minimizar $f(m, b)$ puede expresarse como

$$
f(m, b)=\sum_{i=1}^{N}\left(m x_{i}+b-z_{i}\right)^{2}
$$

y en el caso de la línea ortogonal su expresión es

$$
f(m, b)=\sum_{i=1}^{N} \frac{1}{m^{2}+1}\left(m x_{i}+b-z_{i}\right)^{2}
$$

Si la longitud del perfil se mantiene constante la disminución del parámetro $H / L$ conlleva la disminución de la coordenada $z$ y por tanto de la pendiente de ambas líneas. En las expresiones (6) y (7), se comprueba que cuando $m$ tiende a cero se cumple que $m^{2}$ es mucho menor que $m$ quien a su vez es mucho menor que la unidad, motivo por el que ambas expresiones convergen hacia una misma función.

Tabla III. Valores normalizados de longitud básica y $R a$

\begin{tabular}{lcccccccccc}
\hline Longitud básica (mm) & & & 0.08 & 0.25 & 0.8 & 2.5 & 8 & & \\
\hline $\boldsymbol{R} \boldsymbol{a}(\boldsymbol{\mu m})$ & 0.1 & 0.2 & 0.4 & 0.8 & 1.6 & 3.2 & 6.2 & 12.5 & 25 & 50 \\
\hline
\end{tabular}

Para tener una estimación del orden de magnitud del cociente $H / L$ pueden tenerse en cuenta los valores normalizados de longitudes básicas y de rugosidad media aritmética $R a$ recogidos en la Tabla III. Puede observarse como $H / L$ raramente supera el valor 2.5 encontrándose su rango más frecuente de variación en el intervalo $[0.0005 ; 2.5]$.

\section{Resultados}

Si se representan los valores de los parámetros de rugosidad calculados para el perfil de ejemplo propuesto en el apartado anterior se obtienen los resultados recogidos en la Figura 6. En la figura aparecen los valores de los parámetros $R a$ y $R q$ calculados respecto a las tres líneas de referencia consideradas. Los valores de ordenadas representados son las diferencias de cada parámetro calculado respecto a cada par de líneas de referencia, utilizando como elemento de adimensionalización el valor del parámetro calculado respecto a $L C$, es decir, aparecen de la forma

$$
\frac{R_{L 1}-R_{L 2}}{R_{L C}}
$$

donde $L 1$ y $L 2$ representa un par líneas diferente en cada gráfico y $R$ representa a los parámetros $R a$ y $R q$ según cada gráfica. Se ha elegido un rango de variación de $H / L$ comprendido entre 0 y 0.2 , entendiendo que abarca la mayor parte de las situaciones reales de medida.

Puede comprobarse como la diferencia entre los parámetros disminuye con el parámetro $H / L$ tanto más cuanto más pequeño es éste, independientemente de la línea considerada. Asimismo puede observarse como los gráficos que relacionan los parámetros calculados en las líneas $L C-L M$ y $L C-L O$ son prácticamente idénticos con unos valores ligeramente superiores en el caso de la comparación $L C-L O$. Este hecho queda corroborado en la comparación presentada para las líneas $L M-L O$ donde puede apreciarse como el orden de magnitud es notablemente inferior, del orden de $10^{-5}$ frente a $10^{-4}$ y $10^{-3}$ (según se considere $R a \mathrm{o} R q$ ) de las comparaciones $L C-L M$ y $L C-L O$.

Siguiendo un procedimiento análogo al descrito para el perfil de ejemplo se ha trabajado con unos datos reales obtenidos sobre una serie de 20 probetas cilindradas en un torno de Control Numérico con diferentes valores de avance y velocidad de corte todas ellas medidas en un rugosímetro con palpador inductivo de contacto SM7 tal y como el que aparece en la Figura 2. Los valores obtenidos en las 20 probetas han sido muy similares, habiéndose elegido los correspondientes a una probeta mecanizada con 
avance $f=0.3 \mathrm{~mm} / \mathrm{rev}$ para realizar su representación gráfica. Para la obtención del perfil de rugosidad las medidas se han realizado a lo largo de 5 longitudes básicas de $0,8 \mathrm{~mm}$.
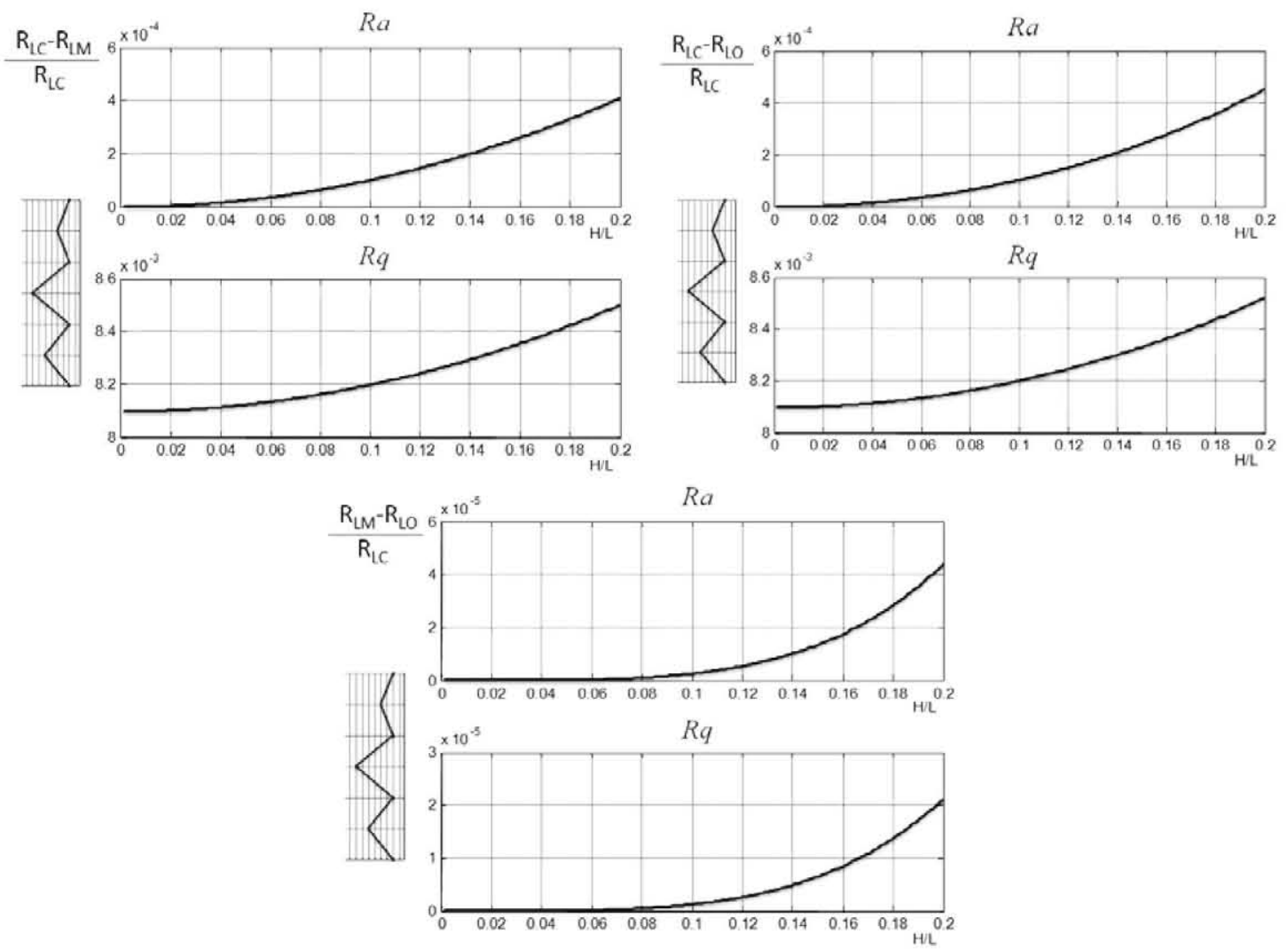

Figura 6. Variación de los parámetros de rugosidad en función de H/L (Perfil Figura3)

En este caso, a diferencia del perfil de ejemplo representado en la Figura 6, debe tenerse en consideración que existen varias longitudes básicas. En la Tabla IV se presentan los valores calculados para el cociente $H / L$ en cada una de las longitudes básicas consideradas. Puede observarse como su valor es muy similar en todas ellas.

Tabla IV. Valores $H / L$ en las longitudes básicas del perfil medido

\begin{tabular}{cccccc}
\hline & $\boldsymbol{l}_{b 1}$ & $\boldsymbol{l}_{b 2}$ & $\boldsymbol{l}_{b 3}$ & $\boldsymbol{l}_{b 4}$ & $\boldsymbol{l}_{b 5}$ \\
\hline$H / L$ & 0.0100 & 0.0098 & 0.0108 & 0.0108 & 0.0103 \\
\hline
\end{tabular}

En la figura 7 se muestra la forma del perfil medido en la que puede apreciarse la huella de la herramienta que ha generado la superficie y el valor del avance empleado $(f=0.3 \mathrm{~mm} / \mathrm{rev})$.

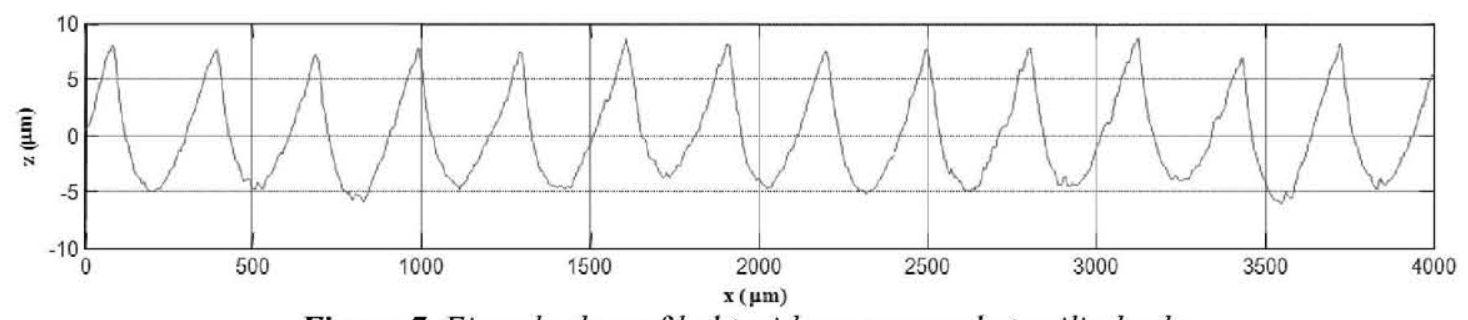

Figura 7. Ejemplo de perfil obtenido en una probeta cilindrada

Los resultados obtenidos sobre las piezas reales están recopilados en la Figura 8. En la representación se ha seguido un criterio análogo al descrito para los datos contenidos en la Figura 6. Tal y como era de esperar los valores obtenidos son considerablemente menores que los estimados en el caso del perfil de prueba, ya que la relación $\mathrm{H} / \mathrm{L}$ es menor en este caso. Es destacable que la diferencia entre los parámetros medidos respecto a la $L M$ y respecto a la $L O$ está en el entorno de $10^{-7}$. 

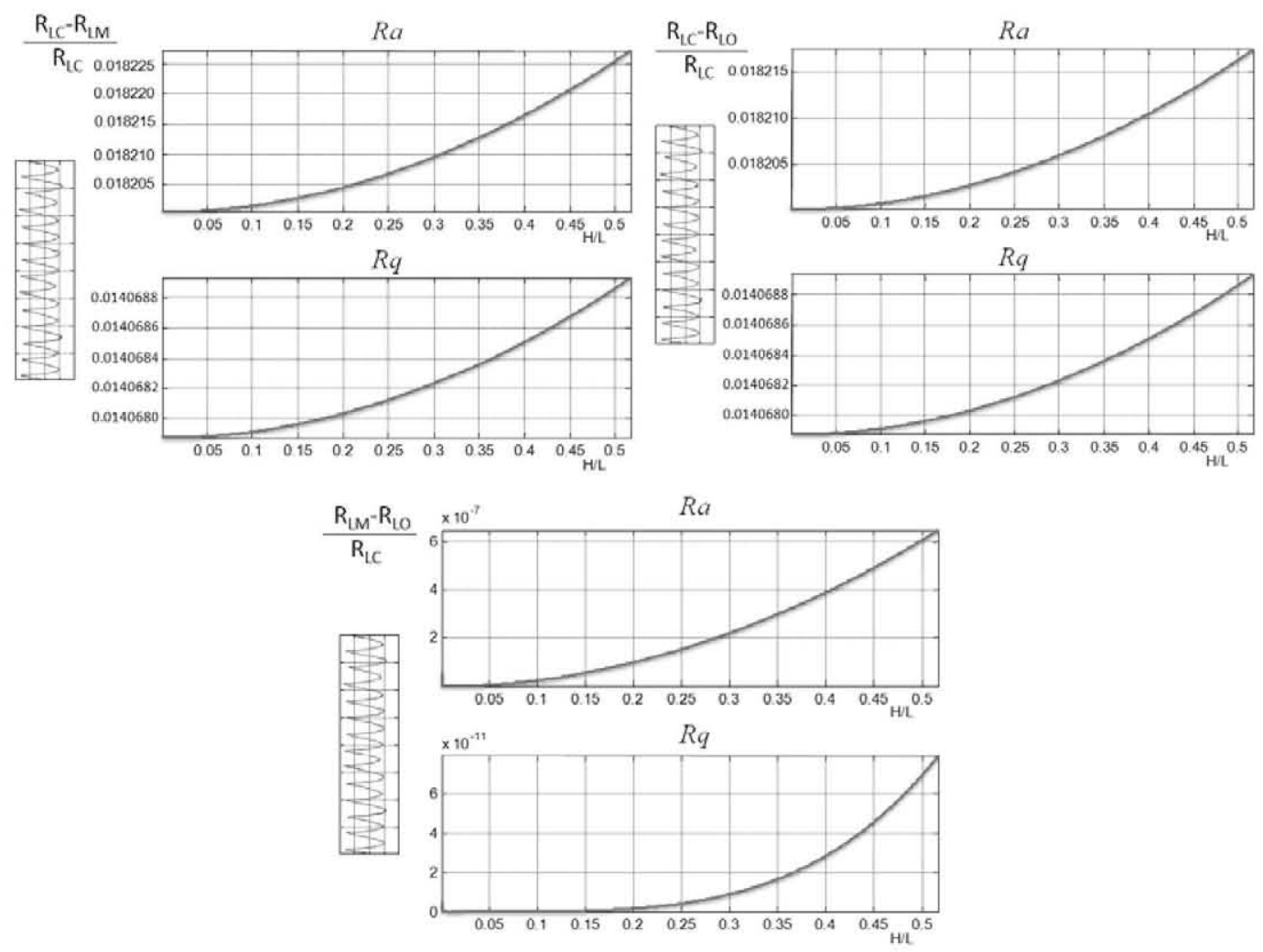

Figura 8. Variación de los parámetros de rugosidad en función de H/L (probetas cilindradas)

\section{Conclusiones}

La caracterización del acabado superficial mediante parámetros numéricos requiere la definición detallada del sistema de referencia respecto al cual se realizan los cálculos. Dentro de este sistema referencia se encuentra la situación del eje de abscisas, definida por la normativa como una línea de mínimos cuadrados, sin una indicación clara respecto a su determinación concreta. En el presente trabajo se ha realizado un estudio acerca de la variación experimentada por los dos principales parámetros de rugosidad $R a$ y $R q$, en función de tres tipos de líneas de mínimos cuadrados, designadas como línea central $L C$, línea media, $L M$ y línea ortogonal $L O$. La primera de ellas, $L C$, es la más sencilla de calcular y se define imponiendo la condición de paralelismo respecto a la dirección de desplazamiento del rugosímetro en la toma de datos. La segunda, $L M$, es la línea de regresión en ordenadas tradicionalmente empleada en otros ámbitos de la ingeniería en los que se requieren ajustes por regresión. La tercera, $L O$, es la menos utilizada a pesar de ser propiamente "la línea de mínimos cuadrados", ya que de todas las posibles líneas que cumplen esta condición es aquélla en la que la suma de los cuadrados de las distancias de los puntos del perfil a ella es mínima. De las tres líneas, la $L O$ es la que genera unos valores menores para una misma información de partida. A la vista de los resultados obtenidos, la principal conclusión es que la utilización de la $L M$ y la $L O$ conduce a los mismos valores calculados en los parámetros $R a$ y $R q$ ya que su diferencia es menor que la resolución habitual de los rugosímetros que está en el orden de $0.01 \mu \mathrm{m}$. También se concluye que para los valores más usuales de la relación entre la altura del perfil y su longitud, la utilización de la línea central es admisible. En cualquier caso siempre que se pueda es conveniente disponer de la información de medida de los puntos del perfil en lugar de confiar sin ninguna otra comprobación en los valores proporcionados por los rugosímetros.

\section{Referencias}

[1] T. R. Thomas, "Rough Surfaces", Imperial College Press, 2nd Edition, ISBN 1-86094-100-1, 1999.

[2] S. Kalpakjian, S. Schmid, "Manufacturing Engineering and Technology", Prentice Hall, 6th Edition, ISBN 0136081681, 2009.

[3] UNE-EN ISO 4287:1999, Especificación geométrica de productos (GPS). Calidad superficial: Método del perfil. Términos, definiciones y parámetros del estado superficial. (ISO 4287:1997+Technical Corrigendum 1).

[4] ASME B46-1-2002. Surface Texture (Surface Roughness, Waviness, and Lay), 2002. 